\title{
The Future of Metabolomics and Individual Monitoring in Antimicrobial Therapy
}

\section{Bueno $J^{*}$}

Research Center of Bioprospecting and Biotechnology for Biodiversity Foundation (BIOLABB) Colombia

Keywords: Metabolomics; Antimicrobial resistance; Anti-infective therapy; Resistome

\section{Introduction}

Antimicrobial drug resistance (AMR) is an eco-evolutionary phenomenon, determined by a pre-existent ability to develop defense mechanisms against aggression by microorganisms combined with a wide spread of antibiotics to nature by humans, this has led to an alteration of microbial populations, whose ecological change leads to one of the greatest problems of public health; in this order of ideas metabolomics is an important tool for to characterize the response of microorganisms and infectious disease to the use of antibiotics; looking for measure the interactome that integrates clinical answer, microbiology, pharmacological design and medicinal chemistry in a personalized model of treatment, which can guarantee the regulation of the biological system comprising anti-infective therapy [1-3]. In this way a interactome of resistome will be the basis for the development of new drugs and prevention of resistance emergence, as well as their evolution with the human holobiont [4,5]. In addition, functional metagenomics can monitor the appearance of resistance genes and spread of plasmid-borne in hosts and clinical environments [6]. The aim of this editorial briefly address antimicrobial resistance from an integral point of view with the ecology and evolution of the individual as a true rational approach that groups the patient, microorganism, environment and antibiotic treatment into a new model of therapy in where the metabolomics is able to offer the greatest number of data with which to make the right decisions both in health care, as well as in public health actions and in reducing the environmental impact of the indiscriminate use of antibiotics.

\section{Metabolomics of Resistome}

The metabolomics of the resistome seeks to establish the different metabolites produced during the emergence of resistance genes, in this route resistant mutants show metabolic alterations in fatty acid composition as well as production of mutaxanthenes, in addition have been possible to determine which types of metabolites are produced by the microbiome during the course of infections as necrotizing enterocolitis $[7,8]$. These biomarkers are not only useful for predicting the clinical outcome to anti-infective therapy; also, it is useful for determining the mechanisms of action of new antibiotics [9].

\section{Synergism}

Multi-drug combinations in optimal doses are the key to current anti-infective therapy, but equally in sub-inhibitory concentrations can be a bad choice if optimization of drug administration is not achieved [10]. In this order of ideas metabolomics can be an important strategy for elucidate the synergistic metabolic pathways that can be selected as antimicrobial targets and optimizing antibiotic pharmacokinetics/ pharmacodynamics that avoid resistance emergence [11].

\section{Biofilms}

Equally, an important microbial factor that promotes AMR is the biofilm formation. For that reason determine biofilm transition and presence will be a fundamental biomarker for select the proper antimicrobial approach. For that reason, detection of Quorum Sensing (QS) molecules that regulates biofilm formation as $\mathrm{N}$-acyl homoserine lactones and Furanosyl borate diester can be useful in early diagnosis and monitoring of bacteria-related diseases [12]. As was demonstrated in cystic fibrosis in which QS signal molecules were detected is sputum, plasma and urine from patients with pulmonary Pseudomonas aeruginosa infection and correlates with clinical status [13].

\section{Metabolomics-on-a-Chip}

An interesting issue in metabolomics topic is the use of metabolomics devices with the ability of detects metabolites that determine metabolic changes in disease process in healthcare settings and with patients under critical condition. In this way microfluidic chip-capillary electrophoresis is the more promising approach for development of devices for on-site analysis and represents an integrative mode for analyte detection, with which to deliver results in time to make decisions in anti-infective treatment [14].

\section{Conclusion}

An eco-evolutionary phenomenon need a multidisciplinary approach, in this way the future of metabolomics is the integration with Omics disciplines, some authors talk about of need for a "permutome" [15]. More necessary than to exchange is to integrate, to form new disciplines that include the tools for the development and study of urgent questions of knowledge, we propose a future of metabolomics more like to "integrome". But is always imperative to remember that the techniques do not integrate, are the problems of public health and crises that make integration the possibility.

\section{Expert Opinion}

In a translational science approach for a rational application of metabolomics in antimicrobial therapy, "integrome" is the choice for obtain more reproducible data encompassing Omics disciplines and tools, as metagenomics that use whole-genome sequencing (WGS) processes which linked to metabolomics can determine the genetic changes that correlate with the secondary metabolites produced both in the health-disease process as in the physiological responses

${ }^{*}$ Corresponding author: Juan B, Research Center of Bioprospecting and Biotechnology for Biodiversity Foundation (BIOLABB), Colombia, E-mail: juanbueno@biolabb.com; juangbueno@gmail.com

Received July 26, 2017; Accepted July 28, 2017; Published August 26, 2017

Citation: Bueno J (2017) The Future of Metabolomics and Individual Monitoring in Antimicrobial Therapy. J Microb Biochem Technol 9: e132. doi:10.4172/19485948.1000e132

Copyright: (c) 2017 Bueno J. This is an open-access article distributed under the terms of the Creative Commons Attribution License, which permits unrestricted use, distribution, and reproduction in any medium, provided the original author and source are credited. 
Citation: Bueno J (2017) The Future of Metabolomics and Individual Monitoring in Antimicrobial Therapy. J Microb Biochem Technol 9: e132. doi:10.4172/1948-5948.1000e132

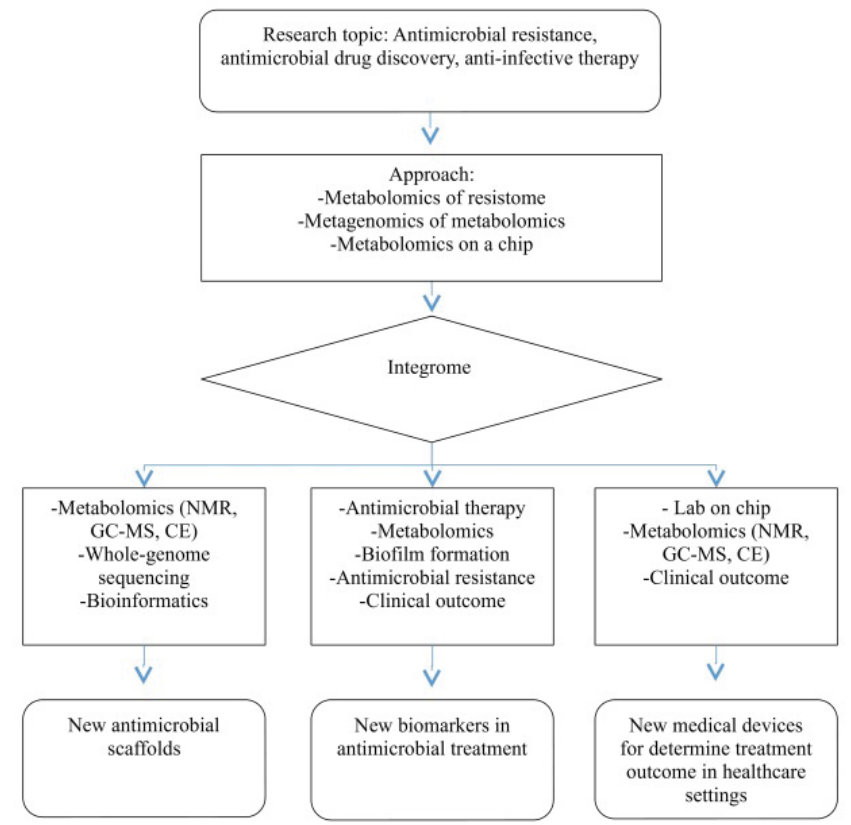

Figure 1: Flowchart of integrome in metabolomics application on antimicrobial therapy.

of microorganisms under the inhibition of their therapeutic targets (Figure 1). In addition, antimicrobial resistance emergence can be monitored with the correlation between resistance genes and secondary metabolites as well as biofilm formation, equally the description of new biomarkers in infectious diseases can determine the effectiveness of anti-infective therapy based on the functionality of metabolic changes. Finally, these advances will be able to develop electronic devices able to support the health personnel in the decision making on site, very useful for early diagnostic of epidemic outbreaks and prevent their spread.

\section{References}

1. Hiltunen T, Virta M, Laine AL (2017) Antibiotic resistance in the wild: An ecoevolutionary perspective. Philos Trans R Soc Lond B Biol Sci 372: 20160039.
2. Zampieri M, Zimmermann M, Claassen M, Sauer U (2017) Non-targeted metabolomics reveals the multilevel response to antibiotic perturbations. Cel Rep 19:1214-1228.

3. Cortese-Krott MM, Koning A, Kuhnle GGC (2017) The reactive species interactome: Evolutionary emergence, biological significance and opportunities for redox metabolomics and personalized medicine. Antioxid Redox Signal.

4. Walsh CT, Wencewicz TA (2014) Prospects for new antibiotics: A moleculecentered perspective. J Antibiot (Tokyo) 67: 7-22.

5. Martí JM, Martínez-Martínez D, Rubio T (2017) Health and disease imprinted in the time variability of the human microbiome. mSystems 2: pii: e00144-16.

6. Crofts TS, Gasparrini AJ, Dantas G (2017) Next-generation approaches to understand and combat the antibiotic resistome. Nat Rev Microbiol 15:422-434.

7. Derewacz DK, Goodwin CR, McNees CR, McLean JA, Bachmann BO (2013) Antimicrobial drug resistance affects broad changes in metabolomic phenotype in addition to secondary metabolism. Proc Natl Acad Sci USA 110: 2336-2341.

8. Wandro S, Osborne S, Enriquez C (2017) Microbial community assembly and metabolite profile of the gut microbiome in extremely low birthweight infants. BioRxiv: 125922

9. Hoerr V, Duggan GE, Zbytnuik L (2016) Characterization and prediction of the mechanism of action of antibiotics through NMR metabolomics. BMC Microbiol 16: 82.

10. Pena-Miller R, Laehnemann D, Jansen G (2013) When the most poten combination of antibiotics selects for the greatest bacterial load: The smilefrown transition. PLoS Biol 11:e1001540.

11. Maifiah MH, Creek DJ, Nation RL (2017) Untargeted metabolomics analysis reveals key pathways responsible for the synergistic killing of colistin and doripenem combination against Acinetobacter baumannii. Sci Rep 7: 45527

12. Zhang B, Powers R (2012) Analysis of bacterial biofilms using NMR-based metabolomics. Future Med Chem 4: 1273-1306.

13. Barr HL, Halliday N, Cámara M, et al. Pseudomonas aeruginosa quorum sensing molecules correlate with clinical status in cystic fibrosis. Eur Respir J 46: 1046-1054

14. Fung YS (2015) Microfluidic chip-capillary electrophoresis: Expanding the scope of application for on-site analysis of difficult samples. In: Fung YS, editor. Microfluidic chip-capillary electrophoresis devices. Boca Raton: CRC press.

15. Livingstone SG, Smith MJ, Silva DS, Upshur RE (2015) Much ado about omics: Welcome to 'the permutome'. J Eval Clin Pract 21: 1018-1021. 